Original Research Article

\title{
Off-label use of antibiotics in hospitalised children in a tertiary care teaching hospital
}

\author{
Puja*, Dhasmana D. C., Saurabh Kohli, Vipin Chander
}

Department of Pharmacology, Himalayan Institute of Medical Sciences, Dehradun, Uttarakhand, India

Received: 22 July 2018 Accepted: 30 August 2018

*Correspondence to: Dr. Puja, Email: sharma_puja_123@ yahoo.co.in

Copyright: () the author(s), publisher and licensee Medip Academy. This is an openaccess article distributed under the terms of the Creative Commons Attribution NonCommercial License, which permits unrestricted noncommercial use, distribution, and reproduction in any medium, provided the original work is properly cited.

\begin{abstract}
Background: Children constitute one-third of the population and they suffer from a variety of infectious diseases and are commonly prescribed antibiotics. Most of the antibiotics lack sufficient information on safety and efficacy in children and are thus prescribed off-label. This study was envisaged to assess the off-label use of antibiotics in hospitalized children.

Methods: Total 120 patients were included in the study. Off-label use was determined on the basis of product literature and National Formulary of India. Descriptive statistics was used to present the data i.e. percentage; proportions, frequency, mean and standard deviation using Microsoft excel worksheet.

Results: A total of 791 drugs from different classes were prescribed to 120 patients with a mean of $6.6 \pm 2.68$ drugs described per patient during their stay in the hospital. 100 out of $120(83 \%)$ patients were prescribed at least one antibiotic during their stay in the hospital. Out of the 204 antibiotics prescribed, 43(21\%) were prescribed off-label. Antibiotic dose was the most common reason followed by age (1month-1 year more than 2-6 years of age) and frequency in off-label use. Conclusions: Antibiotics are commonly prescribed to children with substantial off-label use. The same must be seriously addressed by the policy makers and stakeholders in order to promote their rational use.
\end{abstract}

Keywords: Antibiotics, Hospitalized children, Off-label

\section{INTRODUCTION}

Children constitute about one-third of the total population in developing countries. ${ }^{1}$ On an average a child is prescribed 0.8-3.5 medicines per year on outdoor visits to clinicians inspite of the lack of information on pediatric dosing for most of them. ${ }^{2}$

Most of the drugs do not receive adequate study in infants and children, reasons being the ethical issues related to the inclusion of children in trials, lack of incentives and the reluctance of industry to invest in pediatric studies. As a result, a barrier has been created in making proper drug formulations available for the pediatric patients. Thus, it is difficult to comment on the safety and efficacy of most drugs in children and many drugs are used outside the product license which is termed "off-label". 3

According to World Health Organization (WHO), all "deviations from the conditions of use set forth in the label constitute off-label use. ${ }^{4}$ More elaborately, US Food and Drug Administration (FDA) also describes off-label use of a drug as "Use for indication, dosage form, dose regimen, population or other use parameter not mentioned in the approved labeling." 5

Off-label drug use does not necessarily mean that safety and efficacy have not been investigated at all but scientific evidence falls short of what the drug manufacturers are required to provide to receive approval from regulatory authorities for a particular indication. ${ }^{4}$ Considerable 
problem may be associated with the off-label use in pediatric patients like inefficacy and increased risk of adverse drug reactions.

Epidemiological evaluation of medicine use in elderly has now gained high visibility, but drug prescribing studies in paediatric patients are limited. ${ }^{6}$ India is home to the world's largest child population and children suffer with a variety of diseases which may sometimes be self-limiting or requiring medications. ${ }^{4}$ There is paucity of systematic data in Indian children on the prescription pattern and related off-label use of drugs. The same is true for antibiotics that are most commonly prescribed class of drugs. There have been very few studies on the use of antibiotics in hospitalized children and even lesser on offlabel use of antibiotics in children. So, this study was envisaged to analyse the use of antibiotics in hospitalized children.

\section{METHODS}

This observational cross-sectional study was carried out in Himalayan Institute of Medical Sciences, Swami Ram Nagar, Dehradun, in the Department of Pharmacology and the Department of Pediatrics over a period of twelve months after obtaining ethical clearance from the Institutional Ethics Committee. A total of 120 patients admitted in the pediatric general ward were included in the study.

\section{Inclusion criteria}

Patients less than 18 years of age admitted in pediatric general ward with minimum 5 days of stay in the hospital.

\section{Exclusion criteria}

- Oncological patients and those with chronic diseases

- Patients admitted in pediatric or neonatal intensive care.

\section{Study tools}

- Case recording form

- National Formulary of India, 2011

- Authentic Product label

- World Health Organization's core drug use indicators for rationality of drug use.

\section{Study protocol}

Patient details (age, weight, anthropometric measurements), prescription drug details (indication, dose, frequency) and other relevant information were recorded as per the case recording form after obtaining written informed consent from parent/legal guardian and assent from school going children.

Off-label status of the drug was determined on the basis of National Formulary of India year 2011(NFI)/product literature. The drug was classified off-label on the basis of age if there was lack of information regarding dosing in children or if contraindicated in that particular age. The drug was also classified off-label on the basis of dose if the dose was higher or lower than the dose recommended in the NFI with a $10 \%$ margin. Further, the drug was categorized as off-label if it was used for an indication different than that mentioned in the NFI or product license, or at a different frequency or by a different route of administration.

\section{Statistical analysis}

Descriptive statistics were used to present the data i.e. percentage, proportions, frequency, mean and standard deviation using Microsoft excel worksheet.

\section{RESULTS}

A total of 791 drugs from different classes were prescribed to 120 patients with a mean of $6.6 \pm 2.68$ drugs described per patient during their stay in the hospital. The average duration of stay in the hospital was $8.5 \pm 3.24$ days. The mean age of the patients admitted was $5.5 \pm 5.12$ years. The male to female ratio was 2.42 with a slight preponderance of males.

The most commonly prescribed class of drugs were antimicrobials, followed by drugs acting on the alimentary system and those acting on the nervous system. 100 out of $120(83 \%)$ patients were prescribed at least one antibiotic during their stay in the hospital. 204 antibiotics were prescribed to 100 patients with 38 patients receiving single antibiotic and 62 patients receiving 2 or more than 2 antibiotics (Figure 1).

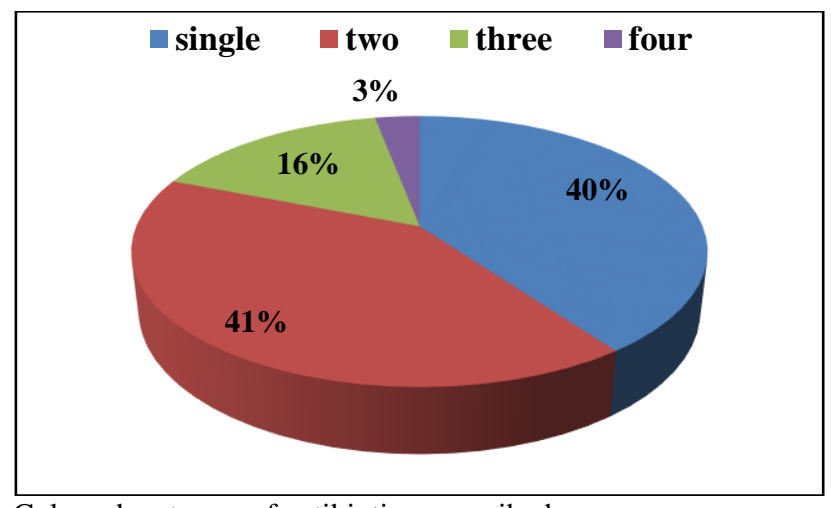

Colour denotes no of antibiotics prescribed

\section{Figure 1: Distribution of patients according to number} of antibiotics prescribed.

Beta-lactams were the most commonly prescribed antibiotic followed by amino glycosides (Figure 2). Out of the 100 patients receiving antibiotic, 91 received antibiotic by parenteral route and remaining 9 patients received antibiotic by oral route.126 (62\%) antibiotics were prescribed by generic name and $78(38 \%)$ were prescribed by brand name. 
Out of the 204 antibiotics prescribed, 43 (21\%) were offlabel use. Dose was the most common reason for off-label use of antibiotics followed by age and frequency (Figure $3)$. off-label use was most common in the age group of 1 month-1 year followed by 2-6 years age group (Figure 4).

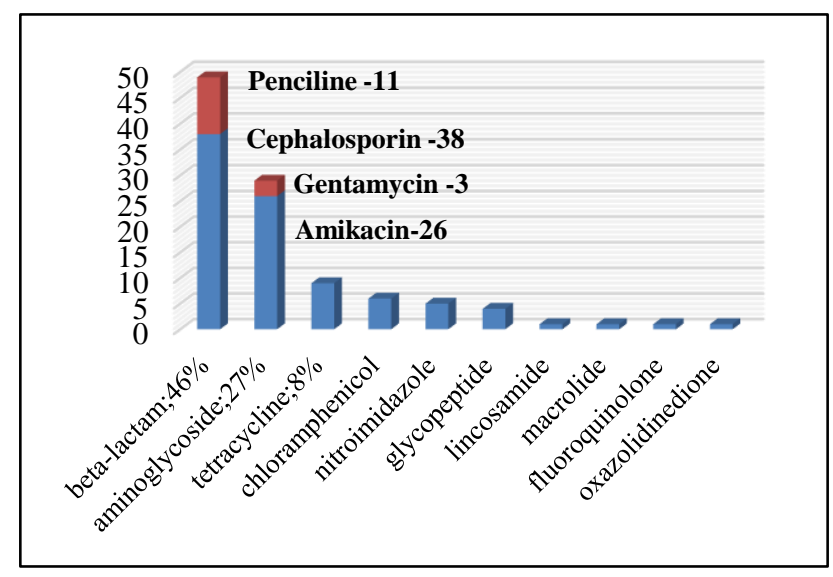

Figure 2: Distribution of groups of antibiotics prescribed to the patients.

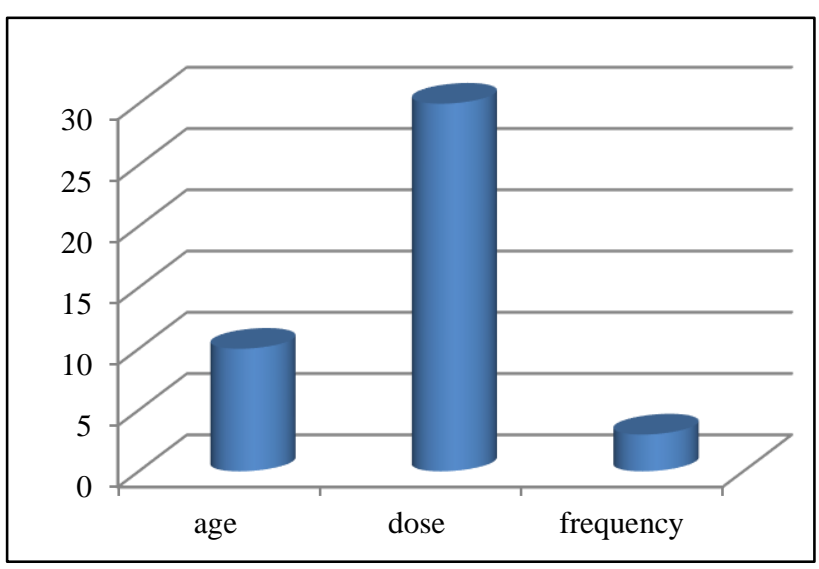

Figure 3: Distribution of drugs according to category of off-label use.

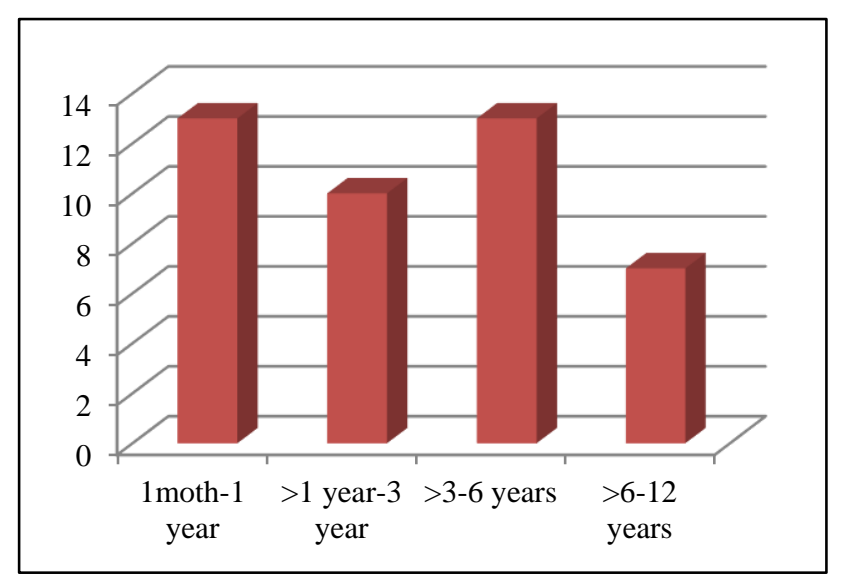

Figure 4: Off- label use of antibiotics in different age groups.
Oral Doxycycline $5 \mathrm{mg} / \mathrm{kg}$ was categorized off-label on the basis of age as it is not recommended for use in children under 8 years of age in the NFI 2011. Ceftriaxone (i.v.) was considered off-label on the basis of dose if it was used at a dose higher or lower than the recommended dose (50$75 \mathrm{mg} / \mathrm{kg}$ ) with a margin of $10 \%$. Similarly, Amikacin was termed off-label on the basis of dose if used at a higher or lower dose than the recommended dose $(15 \mathrm{mg} / \mathrm{kg})$ with $10 \%$ margin.

\section{DISCUSSION}

In this study 791 drugs were administered to a total of 120 patients with a mean of $6.6 \pm 2.68$ drugs per patient. The average number of drugs prescribed per patient was similar to 5.86 as found in study in outdoor patients from a tertiary care center in North India and higher than 3.42 as found in another study from central India in outdoor patients. ${ }^{8,9}$ The minimum number of drugs prescribed was 2 and the maximum number of drugs prescribed was 14 . These findings suggest that polypharmacy was very common and probable lack of common consensus among the clinicians. The most common class of drugs prescribed was antimicrobials $(29.7 \%)$ ). It was similar to $28 \%$ as observed in the study from south India. ${ }^{10}$ Among the antimicrobials most commonly prescribed class of antibiotic was cephalosporins followed by aminoglycosides, penicillins and tetracyclines. Beta lactams were the most commonly prescribed class of antibiotics in the study in hospitalized children from south India. ${ }^{10}$ In this study, most of the patients were hospitalized because of infectitious and parasitic diseases which may be a reason for the high number of antimicrobials prescribed. Among the antibiotics tetracycline (100\%) was prescribed in an offlabel manner on the basis of age as it is contraindicated in children below the age of 12 years to patients diagnosed with scrub Typhus. ${ }^{6}$ Tetracyclines are contraindicated in children because they cause discolouration of the growing enamel in children and affect bone growth. ${ }^{11}$ Cetriaxone and amikacin was prescribed in an off-label on the basis of dose which is similar to that observed in a study on offlabel use. ${ }^{12}$ Ofloxacin and ciprofloxacin were prescribed in one patient each respectively. They were classified as offlabel on the basis of age as these are recommended for use in children because risk of adverse effects like tendon rupture. Linezolid was prescribed to 2 patients and was offlabel on the basis of age as the product insert mentions "safety and efficacy of Linezolid has not been established in children below the age of 15 years. ${ }^{13}$ Gentamicin was prescribed off-label in terms of frequency of administration. About $22 \%$ of the antibiotics prescribed were off-label and more commonly on the basis of age which is similar to $24 \%$ as reported by Portaa A et al, in their study. ${ }^{14}$ Antibiotics are commonly prescribed to children and off-label use of antibiotics is common with many classes of antibiotics not recommended in children where the clinicians have to make prudent decision for the benefit of their patients on the basis of risks and benefits associated. 


\section{CONCLUSION}

This study on prescription pattern and off-label use of drugs in indoor paediatric patients setup creates caveat for further robust studies in this area that can be designed for the benefit of children. More such studies undertaken on a periodic basis with a greater number of a variety of patients and in varied circumstances shall definitely help in understanding the practice of off-label/unlicensed use with their consequences. This may divert the attention of the regulatory authorities and drug industry to invest and explore the paediatric age group drug safety, efficacy and availability of appropriate formulations.

\section{ACKNOWLEDGEMENTS}

Authors would like to thankful to Mr. Shambhu Shankar and all the faculty members at the department of pharmacology at Himalayan institute of medical sciences, Dehradun for their support and motivation.

\section{Funding: No funding sources}

Conflict of interest: None declared

Ethical approval: The study was approved by the Institutional Ethics Committee

\section{REFERENCES}

1. Children in India; 2012. A statistical appraisal social statistics division central statistics office ministry of statistics and programme implementation government of India. Available at: http://www.mospi.children_in_india_2012_rev_pdf_. nic.in

2. Drug treatment. Graeme S Avery. $2^{\text {nd }}$ Edition. Adis press, New York. 1980.

3. Viren B, Montastruc JL, Lapeyere M. Adverse drug reaction and off label drug use in pediatric outpatients. Br J Club Pharma. 2002;54(6):665-70.

4. WHO Drug Information. 2009;23(1). Available at: http://apps.who.int/medicinedocs/en/d/Js17560en/.

5. Conroy S, Choonara I, Impicciatore P, Mohn A, Arnell H, Rane A, et al. Survey of unlicensed and off label drug use in paediatric wards in European countries. BMJ. 2000 Jan 8;320(7227):79-82.

6. Off-label and unlicensed drug prescribing in three paediatric wards in Finland and review of the international literature.

7. Christensen ML, Helms RA, Chesney RW. Is pediatric labeling really necessary?. Pediatrics. 1999 Sep 1;104(Supplement 3):593-7.

8. Kearns GL, Abdel-Rahman SM, Alander SW, Blowey DL, Leeder JS, Kauffman RE. Developmental pharmacology-drug disposition, action, and therapy in infants and children. N Eng J Med. 2003 Sep 18;349(12):1157-67.

9. Ajapuje P, Dhengre P, Giri VC, Khakse GM. Drug prescription practices among paediatric patients in Yavatmal, Central India. Int J Recent Trends Sci and Technology. 2012;5(2):104-6.

10. Fort B. Assessment of drug utilization in hospitalized children at a tertiary care teaching hospital. J Chem Pharmaceut Res. 2014;6(2):592-8.

11. Kline JM, Wietholter JP, Kline VT, Confer J. Pediatric antibiotic use: a focused review of fluoroquinolones and tetracyclines. US Pharm. 2012;37(8):56-9.

12. Sweileh WM. A prospective comparative study of gentamicin-and amikacin-induced nephrotoxicity in patients with normal baseline renal function. Fundamental Clin Pharmacol. 2009 Aug;23(4):51520.

13. Ioannidou M, Apostolidou-Kiouti F, Haidich AB, Niopas I, Roilides E. Efficacy and safety of linezolid for the treatment of infections in children: a metaanalysis. Eur J Pediatr. 2014 Sep 1;173(9):1179-86.

14. Porta A, Esposito S, Menson E, Spyridis N, Tsolia M, Sharland M, et al. Off-label antibiotic use in children in three European countries. Eur J Clin Pharmacol. 2010 Sep 1;66(9):919-27.

Cite this article as: Puja, Dhasmana DC, Kohli S, Chander V. Off-label use of antibiotics in hospitalised children in a tertiary care teaching hospital. Int J Basic Clin Pharmacol 2018;7:1970-3. 